\title{
Some Design and Simulation of Sliding Mode Variable Structure Control for Hopf Bifurcation in Power Systems*
}

\author{
Qiwei $\mathrm{Du}^{1}$, Zhonghua $\mathrm{Su}^{2}$, Sheng $\mathrm{Li}^{2}$ \\ ${ }^{1}$ Zhejiang Electric Power Corporation, Hangzhou, China \\ ${ }^{2}$ School of Electric Power Engineering, Nanjing Institute of Technology, Nanjing, China \\ E-mail: zjsddqw@163.com \\ Received September 11, 2010; revised November 12, 2010; accepted November 15, 2010
}

\begin{abstract}
In power systems, the Hopf bifurcation (HB) can occur before the saddle-node bifurcation (SNB) and becomes one of main reasons of voltage instability and collapse, so the bifurcation control for HB has important significance in improving power system voltage stability. In this paper, the numerical bifurcation analysis software MATCONT was used to study bifurcation behavior of a single-machine dynamic-load (SMDL) system with SVC, and the simulation analysis results show that a unstable Hopf bifurcation (UHB) point occurring before SNB point and engendering potential harm to voltage stability. To delay or eliminate the UHB phenomenon and enhance voltage stability of the SMDL system with SVC, we designed a sliding mode variable structure controller. The switching function and control variables of the controller are clearly described and the derivations are directly provided in detail in this paper. The MATLAB simulation results prove that the designed controller can eliminate the UHB point effectively and ensure safe and stable operation of the system.
\end{abstract}

Keywords: Voltage Stability, Hopf Bifurcation, SMVSC, Simulation

\section{Introduction}

With many voltage instability and collapse accidents occurred in the last 30 years, voltage stability problem has been drawn extensive attention and become a hot spot in power system research. Power systems are complex nonlinear dynamic systems in essence, therefore, the bifurcation theory of nonlinear dynamics has been widely used for studying power system voltage stability problem. Now, saddle-node bifurcation (SNB), limit induced bifurcation (LIB), Hopf bifurcation (HB) and singularity induced bifurcation (SIB) are generally considered to be the main bifurcation types that cause voltage instability and collapse in power systems. HB is one of the typical dynamic bifurcations, when it occurs, the system will produce periodic oscillations which lead to voltage collapse finally. The past researches show that the HB can occur before the SNB and becomes one of main reasons that lead to voltage instability and collapse in power systems [1-5]. Obviously, the bifurcation con-

*Jiangsu Province University Natural Science Research Project of China (08KJD470008) trol aiming at the HB has important significance in improving voltage stability.

Sliding mode variable structure control (SMVSC) is a kind of nonlinear variable structure control strategy, it can act purposefully in the dynamic process according to the current state of the system (such as state variable deviation and its derivative, etc.), which forces the system moving according to the state trajectory of the expected 'sliding mode'. There is no need to design an accuracy object model and online identification system for SMVSC, and with the advantages of fast response and being physically implemented easily, when the system comes into sliding mode motion, it is almost unacted on parameters change and external disturbances. Therefore, SMVSC has a good adaptability and robustness [6,7].

In power system bifurcation analysis, when the $\mathrm{HB}$ occurs, the power system has to take a short time to oscillate before the voltage collapse occurs, and at the same time, SMVSC can make the trajectory of the system reach the designed manifold quickly, and slid the stable equilibrium point. Based on the above analysis, we can realize the control aiming at the HB by using SMVSC method in power systems. 


\section{System Model and Bifurcation Analysis}

\subsection{Power System Model}

A classical single-machine dynamic-load (SMDL) system model was proposed to study voltage stability problem in $[8,9]$. To delay saddle-node bifurcation and improve voltage stability, Reference $[10,11]$ added a static var compensator (SVC) to the load bus in the SMDL system, as shown in Figure 1.

This system in Figure $\mathbf{1}$ can be described by the following differential state equations [10]:

$$
\left\{\begin{array}{l}
\dot{\delta}=\omega \\
\dot{\omega}=\frac{1}{M}\left[P-\frac{E V}{X} \sin \delta-D \omega\right] \\
\dot{V}=\frac{1}{\tau}\left[-k P+V^{2}\left(B-\frac{1}{X}\right)+\frac{E V}{X} \cos \delta\right] \\
\dot{B}=\frac{1}{T}\left(V_{r e f}-V\right)
\end{array}\right.
$$

Where $\delta$ is the generator power angle; $\omega$ is the generator rotor angle speed; $X$ is the line reactance; $E$ is the generator potential; $D$ is the generator damping coefficient; $M$ is the generator moment of inertia; $\tau$ is the time constant of PQ dynamic load; $P$ is the system active power demand; $V$ is the load bus voltage; $k$ is a scalar representing constant power factor of the PQ dynamic load; $B$ is the compensation susceptance of SVC; $T$ is the control time constant of SVC; $V_{\text {ref }}$ is the reference voltage value of load bus. The SVC controller is modeled as a first order pure integrator.

The research results of [10] show that the SVC control can delay SNB point effectively, but also induces a new bifurcation phenomenon - HB in the system.

\subsection{Bifurcation Analysis}

We can use MATCONT, a MATLAB package for numerical bifurcation analysis of ordinary differential equations (ODEs), to analyze bifurcation phenomena of the simple system shown in Figure 1.

The system parameters appearing in (1) are:

$D=0.1$ p.u., $M=0.1 \mathrm{~s}, X=0.5$ p.u., $E=1$ p.u., $k=0.5$, $\tau=0.001 \mathrm{~s}, T=0.01 \mathrm{~s}, V_{\text {ref }}=1 \mathrm{p} . \mathrm{u}$.

The MATCONT bifurcation analysis result for the SMDL system with SVC is depicted in Figure 2. According to Figure 2, a $\mathrm{HB}$ point $(\mathrm{H})$ occurs before SNB point (LP) in the system.

Bifurcation parameter of the $\mathrm{HB}$ point $(\mathrm{H})$ is:

$(\delta, \omega, V, B, P)=(0.785469,0,1,1.293043,1.414314)$.

First Lyapunov coefficient is $6.406133 \mathrm{e}+002(>0)$,

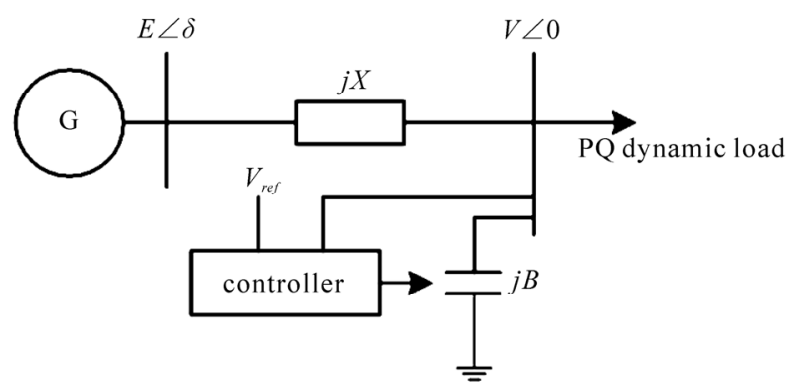

Figure 1. The SMDL system with SVC.

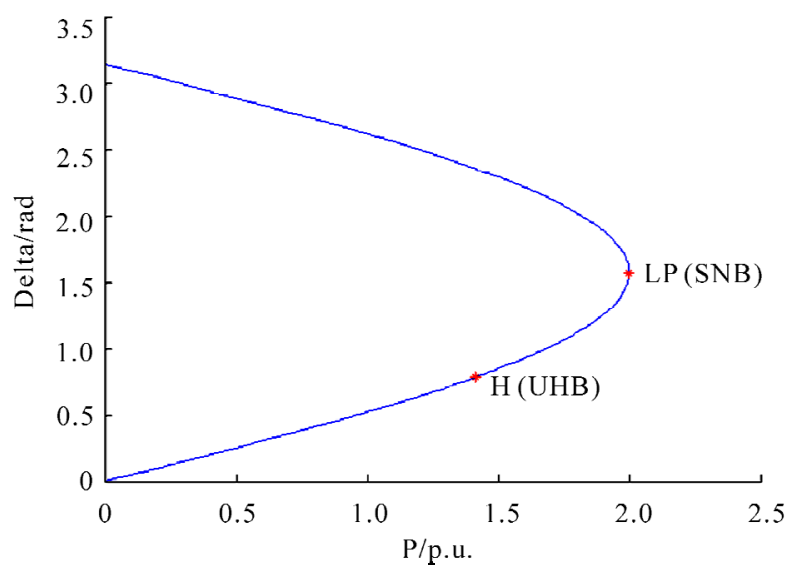

Figure 2. $P$ - $\delta$ curve (based on MATCONT).

so the HB point is a subcritical Hopf bifurcation (namely unstable Hopf bifurcation, UHB), it is harmful to the system voltage stability.

Figure 3 shows the time domain simulation result for a change in $P$ from 1.414314 p.u. to 1.42 p.u. (a small disturbance) at the UHB point. From figure 3 we can know when the UHB occurs, the load bus voltage will lose its stability with the increasing oscillation phenomenon and lead to collapse finally, which can be a mechanism to explain voltage oscillation and instability. Hence, SNB point couldn't be the reference standard of voltage instability, and UHB point can be seen the starting point of voltage instability in power systems.

\section{Design of SMVSC}

It's undoubtedly of great significance to delay or eliminate UHB phenomena in power systems which can improve load limits and enhance voltage stability. References $[12,13]$ designed some new types of SVC controllers based on SMVSC so that the load bus voltage turns more stable. In this section, we will use the SMVSC method to design a SVC controller in the SMDL system, with the purpose of regulating reactive power output of SVC and eliminating or delaying UHB phenomenon. 


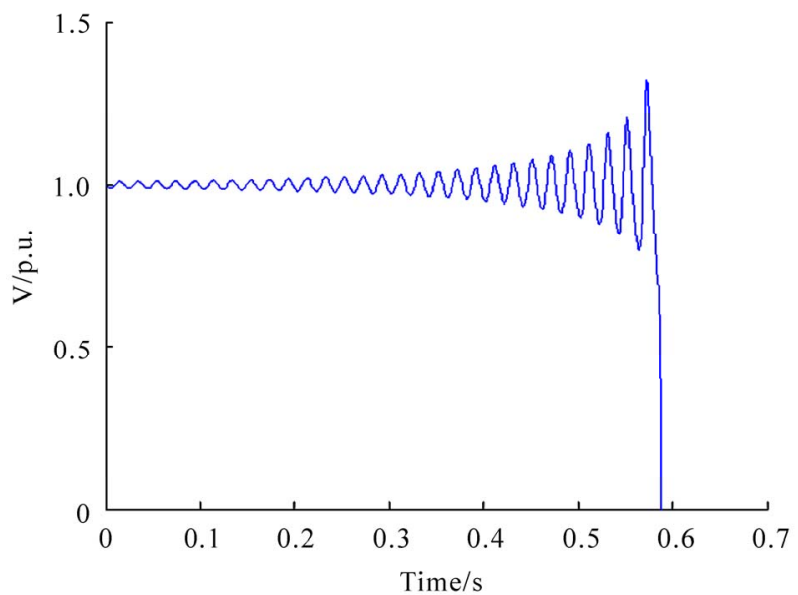

Figure 3. $t$ - $V$ curve (based on MATCONT).

The differential equations describing the system with SMVSC are:

$$
\left\{\begin{array}{l}
\dot{\delta}=\omega \\
\dot{\omega}=\frac{1}{M}\left[P-\frac{E V}{X} \sin \delta-D \omega\right] \\
\dot{V}=\frac{1}{\tau}\left[-k P+V^{2}\left(B-\frac{1}{X}\right)+\frac{E V}{X} \cos \delta\right] \\
\dot{B}=\frac{1}{T}\left(V_{\text {ref }}-V\right)+u
\end{array}\right.
$$

Where $u$ is the control input to the SVC.

Set $x=\left[x_{1}, x_{2}, x_{3}, x_{4}\right]=[\delta, \omega, V, B]$, take $y=h(x)=\delta$ as the output. Change the nonlinear system to the linear system directly:

$$
\left\{\begin{array}{l}
z_{1}=L_{f}^{0} h=h=x_{1}=\delta \\
z_{2}=L_{f}^{1} h=L_{f}\left(L_{f}^{0} h\right)=(\nabla \delta) f=\dot{\delta} \\
z_{3}=L_{f}^{2} h=L_{f}\left(L_{f}^{1} h\right)=(\nabla \dot{\delta}) f=\ddot{\delta} \\
z_{4}=L_{f}^{3} h=L_{f}\left(L_{f}^{2} h\right)=(\nabla \ddot{\delta}) f=\dddot{\delta}
\end{array}\right.
$$

Namely

$$
\left\{\begin{aligned}
z_{1}= & \delta \\
z_{2}= & \dot{\delta}=\omega \\
z_{3}= & \ddot{\delta}=\frac{1}{M}\left(P-\frac{E V}{X} \sin \delta-D \omega\right) \\
z_{4}= & \dddot{\delta}=\frac{1}{M}\left(-\frac{E V \omega}{X} \cos \delta-\frac{D P}{M}+\frac{D E V}{M X} \sin \delta\right. \\
& +\frac{D^{2} \omega}{M}+\frac{k P E}{\tau X} \sin \delta-\frac{E V^{2} B}{\tau X} \sin \delta \\
& \left.+\frac{E V^{2}}{\tau X^{2}} \sin \delta-\frac{E^{2} V}{\tau X^{2}} \sin \delta \cos \delta\right)
\end{aligned}\right.
$$

Suppose $\dot{z}_{4}=\alpha(x)+\beta(x) u$, and make a nonlinear transformation as $u=\beta^{-1}(x)[v-\alpha(x)]$. After derivation calculus to (4), the original system can be translated into the following standard system:

$$
\left[\begin{array}{l}
\dot{z}_{1} \\
\dot{z}_{2} \\
\dot{z}_{3} \\
\dot{z}_{4}
\end{array}\right]=\left[\begin{array}{llll}
0 & 1 & 0 & 0 \\
0 & 0 & 1 & 0 \\
0 & 0 & 0 & 1 \\
0 & 0 & 0 & 0
\end{array}\right]\left[\begin{array}{l}
z_{1} \\
z_{2} \\
z_{3} \\
z_{4}
\end{array}\right]+\left[\begin{array}{l}
0 \\
0 \\
0 \\
1
\end{array}\right] v
$$

In the new coordinates, suppose the switching function of the new system:

$$
S(Z)=C_{1} z_{1}+C_{2} z_{2}+C_{3} z_{3}+z_{4}
$$

where $Z=\left[z_{1}, z_{2}, z_{3}, z_{4}\right]$.

When the system goes into the sliding mode, $S=0$, i.e., $z_{4}=-\left(C_{1} z_{1}+C_{2} z_{2}+C_{3} z_{3}\right)$. Therefore, the sliding mode equations can be given as follows:

$$
\left[\begin{array}{l}
\dot{z}_{1} \\
\dot{z}_{2} \\
\dot{z}_{3}
\end{array}\right]=\left[\begin{array}{ccc}
0 & 1 & 0 \\
0 & 0 & 1 \\
-C_{1} & -C_{2} & -C_{3}
\end{array}\right]\left[\begin{array}{l}
z_{1} \\
z_{2} \\
z_{3}
\end{array}\right]
$$

The eigenvalue equation of (7) is:

$$
\lambda^{3}+C_{3} \lambda^{2}+C_{2} \lambda+C_{1}=0
$$

Supply the poles $\left[k_{1}, k_{2}, k_{3}\right]$, which enable the sliding mode Equations (7) to be stable in advance, then $\left[C_{1}, C_{2}, C_{3}\right]$ are derived:

$$
\left\{\begin{array}{l}
C_{1}=-k_{1} k_{2} k_{3} \\
C_{2}=k_{1} k_{2}+k_{2} k_{3}+k_{1} k_{3} \\
C_{3}=-\left(k_{1}+k_{2}+k_{3}\right)
\end{array}\right.
$$

Substitute the nonlinear transfer equations $Z=T(x)$ (namely (4)) for the switching function $S(Z)$, then the switching function of the system in the original coordinates can be derived.

Let $\dot{S}$ equal the exponential reaching law:

$$
\dot{S}=-\varepsilon \operatorname{sgn} S-l S
$$

Where $\varepsilon>0$ and $l>0$, then

$$
-\varepsilon \operatorname{sgn} S-l S=C_{1} z_{2}+C_{2} z_{3}+C_{3} z_{4}+v
$$

So $v=-(\bar{C} Z+\varepsilon \operatorname{sgn} S+l S), \quad \bar{C}=\left[0, C_{1}, C_{2}, C_{3}\right]$, and now $u$ can be derived as follows:

$$
\begin{aligned}
u= & \beta^{-1}(x)[v-\alpha(x)] \\
= & -\beta^{-1}(x)[\bar{C} Z+\varepsilon \operatorname{sgn} S+l S+\alpha(x)] \\
= & -\beta^{-1}(x)[\bar{C} T(x)+\varepsilon \operatorname{sgn} S(T(x)) \\
& +l S(T(x))+\alpha(x)]
\end{aligned}
$$

Now we have finished designing the switching function and the law of the SMVSC. In the next section, the simu- 
lation analyses about control effect of the designed controller will be introduced.

\section{Time Domain Simulation}

To observe and study control effect of the designed variable structure controller, a MATLAB-procedure was written and executed for the time domain simulation analyses. The flow chart of extracting the control variable $u$ is depicted in Figure 4.

According to the previous analyses based on MATCONT, we can know that in the SMDL system with SVC, the UHB occurs when bifurcation parameter is: $(\delta, \omega, V$, $B, P)=(0.785469,0,1,1.293043,1.414314)$.

Make the UHB point be the initial state of the SMDL system with SVC, when the system is subjected to a small disturbance ( $P$ is from 1.414314p.u. to 1.42p.u.), the time domain simulation results without SMVSC and with SMVSC are depicted in Figure 5 and Figure 6 respectively. According to Figures 5-6, conclusions below can be got:

1) When the system without SMVSC operates at the HB point, after the small disturbance occurs, the load bus voltage will have a sudden drop at about $0.57 \mathrm{~s}$ after the increasing oscillation, and at the same time, the generator power angle also loses its stability.

2) When the system with SMVSC operates at the HB point, after the small disturbance occurs, the load bus voltage won't have an oscillation and keeps stable, and at the same time, the generator power angle also keeps its stability.

3) The load bus voltage after variable structure control has a little erratic swing at all time, this is because the

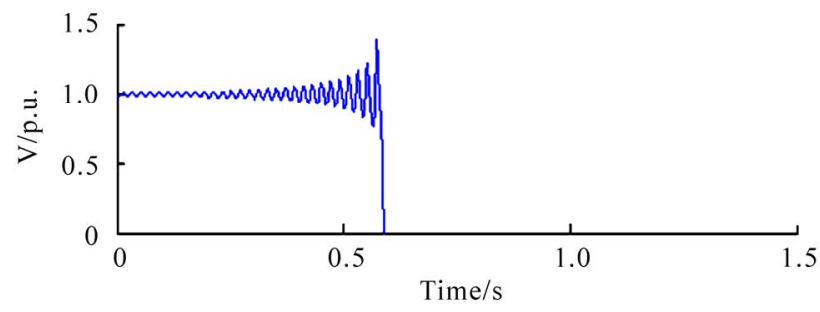

SMVSC is very complex and it keeps regulating the load bus voltage. However, the swing amplitudes are very small, and the voltage can remain in the qualified area.

\section{Conclusions}

In this paper, a simple but meaningful and representative power system model, the single-machine dynamic-load system with SVC, was used to analyze Hopf bifurcation and voltage stability. We also used the SMVSC method to design a controller to stabilize the load bus voltage and control UHB phenomenon of the simple system by controlling the reactive power output of the SVC. Simu-

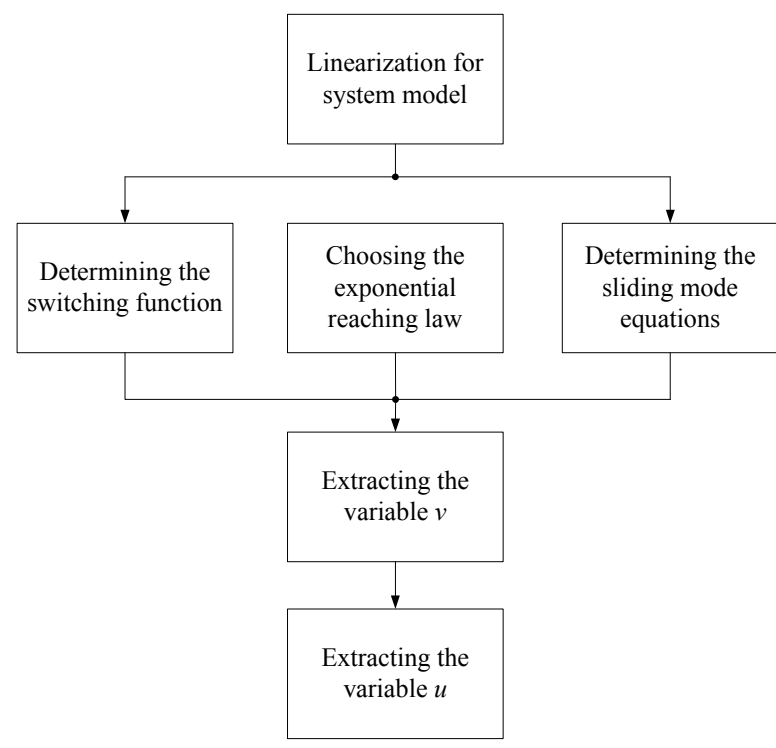

Figure 4. Flow chart of extracting the variable $u$.

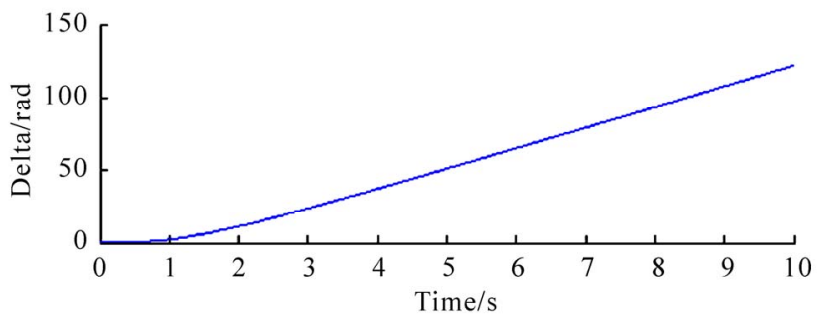

Figure 5. Time domain simulation results without SMVSC.
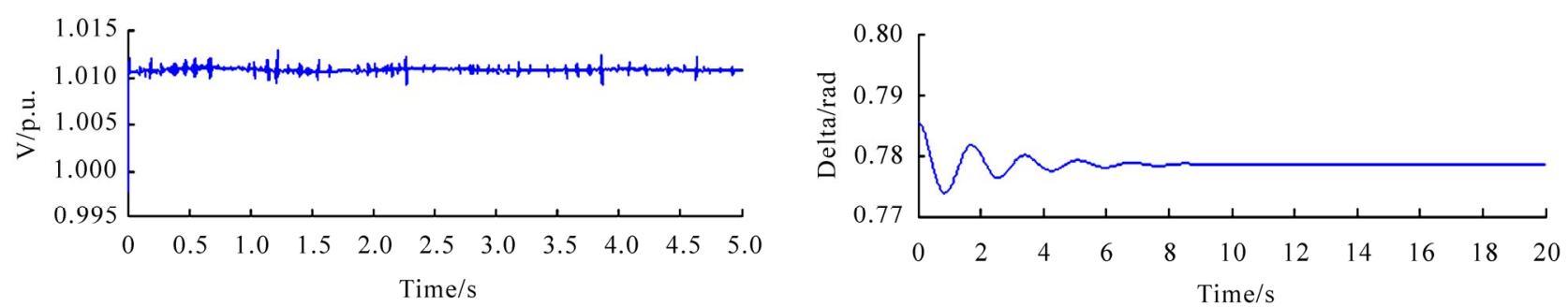

Figure 6. Time domain simulation results with SMVSC. 
lation results show that the UHB of the original system can be eliminated effectively and the voltage stability margin is improved.

The SMVSC method can eliminate the UHB, but also bring some new problems. For example, we can derive the voltage stability index and the voltage stability margin of the original system by the methods that have been found in $[14,15]$, but there is no method for the system with SMVSC at present. In addition, the structure of the SMVSC is very complex, and it's very difficult to change the controller into a practical product now. These problems will take a long time to be studied. Therefore, the SMVSC method in many areas including power systems is still in the stage of theoretical research. There is an urgent need to solve above problems depending on a variety of advanced automatic control technologies.

\section{References}

[1] Y. Wang, H. Chen, R. Zou, "A Nonlinear Controller Design for SVC to Improve Power System Voltage Stability," Electrical Power and Energy Systems, Vol. 22, No. 7, October 2000, pp. 463-70. doi:10.1016/S0142-0615 (00)00023-5

[2] Z. J. Jing, D. S. Xu, Y. Chang, and L. L. Chen, "Bifurcations, Chaos, and System Collapse in a Three Node Power System," Electrical Power and Energy Systems, Vol. 25, No. 6, July 2003, pp. 443-61. doi:10.1016/S014 2-0615(02)00130-8

[3] S. S. Mohamed, A. H. Munther, H. A. Eyad and E. Abdel-Aty, "Delaying Instability and Voltage Collapse in Power System Using SVCs with Washout Filter-Aided Feedback," Proceedings of 2005 American Control Conference, Portland, 2005, pp. 4357-4362.

[4] S. F. Liu, J. F. Gao and P. Li, "Multi-Parameter Bifurcation Analysis of a Typical Power System with the Walve Aggregated Load Model," Relay, Vol. 32, No. 19, 2004, pp. 13-17.
[5] Z. W. Peng, G. G. Hu and Z. X. Han, "Power System Voltage Stability Analysis Based on Bifurcation Theory," China Electric Power Press, Beijing, 2005.

[6] Y. M. Hu, "Variable Structure Control Theory and Application," Science Press, Beijing, 2003.

[7] W. B. Gao, "Variable Structure Control Theory and Design Methods," Science Press, Beijing, 1996.

[8] C. A. Caňizares, "On Bifurcation, Voltage Collapse and Load Modeling," IEEE Transactions on Power Systems, Vol. 10, No. 1, 1995, pp. 512-522. doi:10.1109/59.37397 8

[9] C. A. Caňizares, "Calculating Optimal System Parameters to Maximize the Distance to Saddle-Node Bifurcations," IEEE Transactions on Circuits and Systems-I, Vol. 45, No. 3, 1998, pp. 225-237. doi:10.1109/81.662696

[10] W. Gu, F. Milano, P. Jiang and G. Q. Tang, "Hopf Bifurcations Induced by SVC Controllers: A Didactic Example," Electric Power Systems Research, Vol. 77, No. 3-4, pp. 234-240, 2007. doi:10.1016/j.epsr.2006.03.001

[11] S. Li and Z. H. Su, "Static State Feedback Control for Saddle-Node Bifurcation in a Simple Power System," Proceedings of the 2nd Conference on Power Electronics and Intelligent Transportation System, Shenzhen, Vol. 1, 2009, pp. 158-160.

[12] J. W. Du, B. Wang and W. Cai, "Study on Nonlinear Grey Sliding Mode Controller for Static Var Compensator," Electrotechnical Application, Vol. 26, No. 7, pp. 5053, 2007.

[13] Z. J. Kang and F. Y. Meng, "Nonlinear Variable Structure SVC Control Based on Extended States Observer," Relay, Vol. 35, No. 22, 2007, pp. 10-13.

[14] Y. Li, Y. J. Zhang and W. Liu, "Fast Determination of Voltage Stability Critical Point and Its Sensitivity Algorithm," Power System Technology, Vol. 32, No. 18, 2008, pp. 47-51.

[15] B. Dai, J. H. Zhang, and J. Liu, "Influence of Load Increase Pattern on the Oscillatory Instability Margin of Power Systems," Proceedings of the CSEE, Vol. 28, No. 25, 2008, pp. 44-49. 